\title{
Relation between Toxicity of Cryoprotectant DMSO and Its Concentration in Several Fish Embryos
}

\author{
Toru Suzuki, ${ }^{* 1}$ Hiroaki Komada, ${ }^{* 1}$ Rikuo Takai, ${ }^{* 1}$ Keiji Arii, ${ }^{* 2}$ and Tsuneo T. Kozima*3 \\ ${ }^{*}$ Department of Food Science and Technology, Tokyo University of Fisheries, Konan, Minato, Tokyo 108, Japan \\ ${ }^{*}$ Daikin Kogyo Ltd., Kaneoka, Sakai, Osaka 591, Japan \\ ${ }^{*}$ Department of Food Science, College of Bioindustry, Tokyo University of Agriculture, Yasaka, Abashiri, Hokkaido \\ 099-24, Japan
}

(Received June 3, 1994)

\begin{abstract}
Although dimethyl sulfoxide (DMSO) has been often used in cryopreservation of fish embryo as an effective cryoprotectant, its maximum concentration in terms of its toxicity was obscure. When the embryos of several fish species, medaka, pejerrey, rainbow trout and carp, were immersed in solutions of various DMSO concentrations, the amounts of DMSO penetrating into the embryos were measured by HPLC and simultaneously their mortalities were determined from observation under microscope. From the uptake curves of DMSO, the permeability coefficients of DMSO to the egg membrane were estimated to be 0.13 to $1.09 \times 10^{-4} \mathrm{~cm} / \mathrm{sec}$. Furthermore, the relations of accumulative mortality v.s. DMSO concentration in embryos were well expressed by a cumulative normal distribution function, and the average DMSO lethal concentration of each embryo, giving a cumulative mortality of $50 \%$, and its variance were determined. It was concluded that the tolerance to DMSO becomes higher in the order of carp, rainbow trout, pejerrey, and medaka.
\end{abstract}

Key words: cryoprotectant, DMSO, fish embryo, toxicity, rainbow trout, medaka, pejerrey, carp

Some researches ${ }^{1-6)}$ on the cryopreservation of fish embryos and eggs have been attempted, but a successful longtime cryopreservation method has not yet been established. It is known that the cryopreservation of small cells such as mammalian embryos ${ }^{7)}$ and sperms ${ }^{8)}$ and fish sperm $^{9,10)}$ is possible and is actually used for preservation for long periods. Usually, for the cryopreservation of small cells, cryoprotectants such as dimethyl sulfoxide (DMSO), glycerol and saccharides are used to immerse the cells in the cryoprotectant solution. The cryoprotectants are thought to penetrate into the cells and help to vitrify substances in the cell ${ }^{11)}$ without fatal intracellular freezing. In general, the higher the intracellular concentration of cryoprotectants, the easier the vitrification becomes. Therefore, it is desirable that the intracellular concentration of cryoprotectant is high. On the other hand, most cryoprotectants have a toxic effect on cells to a certain extent. DMSO, which is one of the most effective cryoprotectants, is also known to be harmful for some cells. ${ }^{12)}$ As a first step to successful cryopreservation, it is important to determine the optimum intracellular concentration of cryoprotectant.

There is no difference in principle between the cryopreservation of fish eggs or embryos and other animals. However, since fish eggs and embryos are large and have a hard, thick membrane compared with other cells, there are difficulties not only for heat transfer during cooling but also for penetration of the cryoprotectants into the eggs. ${ }^{13)}$ We believe that it is necessary to understand precisely the relation of the penetration and toxicity of cryoprotectants to fish eggs or embryos. Nevertheless, for fish embryos and eggs, there have been few quantita- tive investigations on the penetration of cryoprotectants into the embryos or eggs and their toxicity. Especially, the maximum concentration of cryoprotectant in terms of toxicity is still not clear. Although Harvey et al. ${ }^{14)}$ reported the uptakes of labeled glycerol and DMSO by the developing embryo of the zebra fish, they could not show the toxicity of the cryoprotectants because the immersion tests were conducted at low concentrations of cryoprotectant. In our previous report, ${ }^{15)}$ we compared the mortality time to inner DMSO concentration of rainbow trout embryos after immersing the embryos in DMSO solution, and suggested that the mortality of rainbow trout embryos could be observed when the DMSO concentration in the embryos was higher than a critical value. However, in that study, the DMSO concentration in the embryos was calculated by assuming the egg membrane permeability coefficient of DMSO since we could not actually measure the DMSO concentrations.

This study evaluates quantitatively the toxicity of cryoprotectant to several fish embryos related to its penetration into the embryos. Using DMSO as one of the most popular cryoprotectants, immersion tests in solutions containing various high DMSO concentrations were carried out. During immersion, the mortalities were measured and simultaneously the amounts of DMSO penetrated in the embryos were determined by using HPLC. These results on the permeability of DMSO to egg membranes and the average lethal DMSO concentration are discussed.

\section{Materials and Methods}

Embryos of four freshwater fish species, rainbow trout 
Salmo Gairdneri, pejerrey Odonthestes Bonariensis, carp Cyprinus Carpio and medaka Oryzias Latipes, were used. The embryos of rainbow trout, pejerrey and carp were provided by the Department of Aquatic Biosciences, Tokyo Univ. of Fisheries. The embryos of rainbow trout and pejerrey were at the eyed stage. Carp embryos were at the morula stage. We started our experiments without storing them after transfer to our laboratory. The medaka embryos of the eyed stage were taken from stocks at our laboratory just before use.

The cryoprotectant, DMSO ( $>98.5 \%)$ and the chemicals for $\mathrm{HPLC}$ use, $\mathrm{HClO}_{4}(60 \%)$ and $\mathrm{H}_{2} \mathrm{SO}_{4}(95 \%)$ were purchased from Wako Pure Chemical Industries, Ltd. Distilled water was used without further purification. DMSO solutions of various concentrations ranging from 2 to $5 \mathrm{M}$ were prepared just before the immersion procedure, and were dispensed in $75 \mathrm{ml}$ aliquots into dishes. All sample were run in duplicate.

The embryos of every species were divided into two groups, and after the same immersion procedure one group was used for mortality observations while the other was used for determining the concentration of DMSO penetrating into the embryos.

\section{DMSO Immersion Procedure}

About 20 embryos were transferred to each petri dish which was filled with a DMSO solution of a given concentration and maintained for $120 \mathrm{~min}$ at room temperature. Simultaneously, the same procedures were carried out using DMSO solutions of different concentrations. The immersion conditions and the character of the examined embryos are listed in Table 1 . The diameters of 20 embryos were measured under a microscope just before immersion, and the average diameters of embryos were determined. The volumes and specific surface areas were calculated by assuming the embryos to be spherical. The temperature of the solutions during immersion was kept at $11^{\circ} \mathrm{C}$ for the rainbow trout embryos and at $20^{\circ} \mathrm{C}$ for the other embryos.

\section{Mortality Measurement}

After a given immersion time, the number of dead embryos were counted under a microscope. The cumulative mortality was defined as the ratio of the number of dead embryos to that of initial embryos. Death of the embryos except for the carp embryos was judged from lack of cardiac movement. In the case of the carp embryos, we judged the embryo to be dead if the morula stage did not develop to the next stage during incubation in water after DMSO immersion.

DMSO Concentration in Embryos ${ }^{16)}$

Several embryos were removed from the DMSO solu-

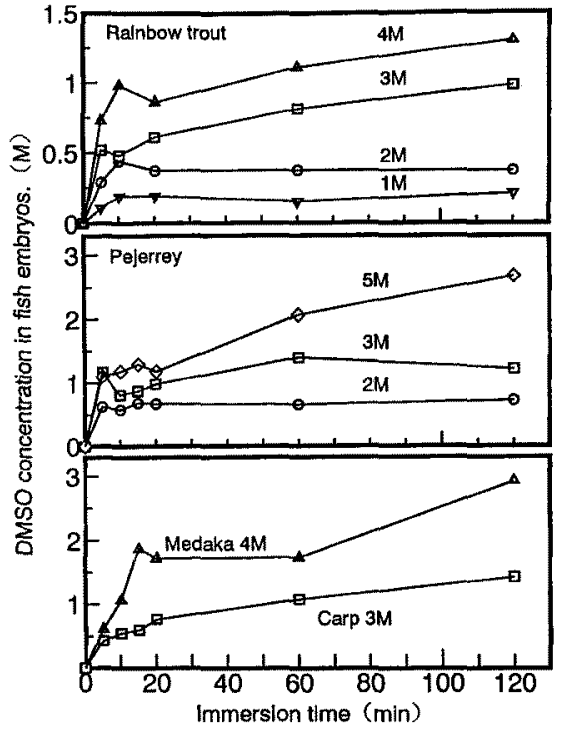

Fig. 1. Changes in DMSO concentrations in embryos of four fish species during immersion into solutions of various DMSO concentrations; $\nabla, 1 \mathrm{M} ; 0,2 \mathrm{M} ; \square, 3 \mathrm{M} ; \Delta, 4 \mathrm{M} ; 0,5 \mathrm{M}$.

tion at selected intervals. Each embryo was homogenized with $5 \mathrm{ml}$ of $5 \% \mathrm{HClO}_{4}$ for protein precipitation after washing with a small amount of $0.15 \mathrm{M}$ saline on gauze. The homogenates were passed through a $0.45 \mu \mathrm{m}$ membrane filter and a $5 \mu l$ portion was injected into an HPLC system as described below.

The HPLC system consisted of a Bio-Rad Aminex HPX-87H column $(7.8 \times 300 \mathrm{~mm})$, Shimadzu LC-6A pump, CTO-6A column oven at $35^{\circ} \mathrm{C}$, SPD-6A UV detector at $210 \mathrm{~nm}$, and Chromatopac C-R6A. The mobile phase was $0.003 \mathrm{M} \mathrm{H}_{2} \mathrm{SO}_{4}$ and the flow rate was set at 0.6 $\mathrm{ml} / \mathrm{min}$. The DMSO concentration in the embryo, which was defined as the number of mol per embryo volume, was determined and calculated from an authentic DMSO solution.

\section{Results and Discussion}

\section{DMSO Concentration in Embryos}

All HPLC chromatograms gave sharp and separable DMSO peaks (figures not shown), so the DMSO concentrations in the embryos could be calculated from the peaks. No previous HPLC reports have been used to determine the DMSO concentration in fish embryos or eggs. This technique was more convenient than the isotope labeling method used by Harvey et al. ${ }^{14)}$

Figure 1 shows the changes of DMSO concentrations in

Table 1. The immersion condition and the character of the examined embryos

\begin{tabular}{lccccc}
\hline Sample & Stage & $\begin{array}{c}\text { Average } \\
\text { diameter }[\mathrm{cm}]\end{array}$ & Volume $\left[\mathrm{cm}^{3}\right]$ & $\begin{array}{c}\text { Specific surface } \\
\text { area }\left[\mathrm{cm}^{2} / \mathrm{cm}^{3}\right]\end{array}$ & $\begin{array}{c}\text { DMSO concentration of } \\
\text { immersion solution }[\mathrm{M}]\end{array}$ \\
\hline Rainbow trout & eye & $0.5 \pm 0.05$ & 0.0654 & 12.0 & $1,2,3,4$ \\
Pejerrey & eye & $0.14 \pm 0.01$ & 0.0014 & 42.9 & $2,3,5$ \\
Medaka & eye & $0.14 \pm 0.005$ & 0.0014 & 42.9 & 4 \\
Carp & morula & $0.14 \pm 0.01$ & 0.0014 & 42.9 & 3 \\
\hline
\end{tabular}


embryos by the immersion procedure. The DMSO concentrations in the embryos of every species significantly increased for $10 \mathrm{~min}$ after they were immersed in the DMSO solution. The concentration then decreased slightly from 10 to $20 \mathrm{~min}$, and gradually increased again to each equilibrium concentration. The reasons for this transient decrease of DMSO concentration in the embryos which was significant with higher DMSO concentration in the immersion solution are not clear at this stage. As a possible explanation, the outflow effect of water through the egg membrane by osmotic pressure during the initial period of immersion, i.e., shrinking of the embryo volume, may be considered. Arii et al. ${ }^{17)}$ reported the shrinking of medaka embryo during immersion in DMSO solution, however, they did not determine the DMSO concentration in the embryo. In this present report, we calculated the concentration assuming that the volume of the embryo does not change during the experiment. Namely, the DMSO concentrations in Fig. 1 correspond to the absolute DMSO contents in each embryo irrespective of volume change of embryo. Therefore, this kind of response on DMSO concentration is thought to be independent of osmotic effects. Even Harvey, ${ }^{14)}$ who measured the penetration of DMSO into fish embryos, did not describe this effect. Since DMSO concentrations used for usual immersion tests are within $2 \mathrm{M}$, the response may be overlooked. More detailed investigation is necessary to clarify cause.

\section{Egg Membrane Permeability Coefficient of DMSO}

We also determined the permeability coefficient $k$ of DMSO to the egg membrane from the initial gradients of each DMSO uptake curve in Fig. 1. We assumed that the penetrating velocity depended on the difference of cryoprotectant concentration between the inside of the egg and outside of it, the surface area of the egg and permeablity coefficient. This is given by the following equation:

$$
\left(\mathrm{d} C_{\mathrm{in}} / \mathrm{d} t\right)=k(A / V)\left(C_{\text {out }}-C_{\mathrm{in}}\right) .
$$

This equation expresses the transport rate of DMSO through the egg membrane into the embryo. $C_{\text {in }}$ and $C_{\text {out }}$ are the DMSO concentrations in the embryo and in the immersion solution, respectively. $A$ and $V$ are the surface area and volume of the embryo, respectively. At the initial

Table 2. The estimated permeability coefficient $k$ of DMSO of the egg membranes of several fish embryos

\begin{tabular}{lcccc}
\hline \multicolumn{1}{c}{ Sample } & $\begin{array}{c}C_{\text {out }} \\
{[\mathrm{M}]}\end{array}$ & $\begin{array}{c}\mathrm{d} C_{\mathrm{in}} / \mathrm{d} t \\
{[\mathrm{M} / \mathrm{sec}]} \\
(\mathrm{at} t=0)\end{array}$ & $\begin{array}{c}k \\
(\mathrm{~cm} / \mathrm{sec})\end{array}$ & $\begin{array}{c}\text { Tested } \\
\text { temperature } \\
{\left[{ }^{\circ} \mathrm{C}\right]}\end{array}$ \\
\hline Rainbow trout & & & & \\
& 1 & 0.0008 & $0.67 \times 10^{-4}$ & \\
& 2 & 0.0021 & $0.87 \times 10^{-4}$ & 11 \\
& 3 & 0.0037 & $1.04 \times 10^{-4}$ & \\
Pejerrey & 4 & 0.0053 & $1.09 \times 10^{-4}$ & \\
& 2 & 0.0032 & $0.37 \times 10^{-4}$ & \\
Medaka & 3 & 0.0059 & $0.46 \times 10^{-4}$ & 20 \\
Carp & 5 & 0.0055 & $0.26 \times 10^{-4}$ & \\
& 4 & 0.0024 & $0.14 \times 10^{-4}$ & 20 \\
\hline
\end{tabular}

stage of immersion, $C_{\mathrm{in}}$ is approximately 0 . Therefore, if the initial gradient of the DMSO uptake curve, i.e. $\left(\mathrm{d} C_{\mathrm{in}} /\right.$ $\mathrm{d} t$ ) within a few minutes, is known, the permeability coefficient $k$ can be estimated.

As shown in Table 2, the permeability coefficient $k$ of DMSO to the egg membrane of the examined embryos ranged from $0.13 \times 10^{-4}$ to $1.09 \times 10^{-4} \mathrm{~cm} / \mathrm{sec}$. These values are small compared with the value $\left(2.25 \times 10^{-4} \mathrm{~cm} /\right.$ sec) of other cells published in the literature. ${ }^{12)}$ This is thought to be a reasonable result because the egg membrane of fish is known to be hard and thick. Although the permeability coefficient of DMSO for rainbow trout increased with DMSO concentration in the immersion solution, this increase is thought to be of no significance because the degree of increase is within the range of error of estimating the permeability coefficient. It should be noted that the figures of the permeability of DMSO listed in Table 2 can be used to estimate the DMSO concentration in fish embryos for cryopreservation.

From a comparison of the DMSO uptakes among the embryos, the inner DMSO concentrations in the rainbow trout embryo were much lower than those of the other species. This is probably due to the difference in embryo size as listed in Table 1. The average diameter of rainbow trout embryos was much larger than the others, despite the high permeability of DMSO to the egg membrane of the rainbow trout as shown in Table 2. Therefore, the inner DMSO concentration increases more slowly.

\section{Cumulative Mortality}

In the mortality test of the eyed stage embryos, as the immersion time proceeded the pectoral fin showed convulsive movement, the blood stream stopped, and at last cardiac movement stopped. Fatal carp embryo of the morula stage became cloudy as the immersion time increased and development to the eyed stage was not recognized during incubation in water after immersion.

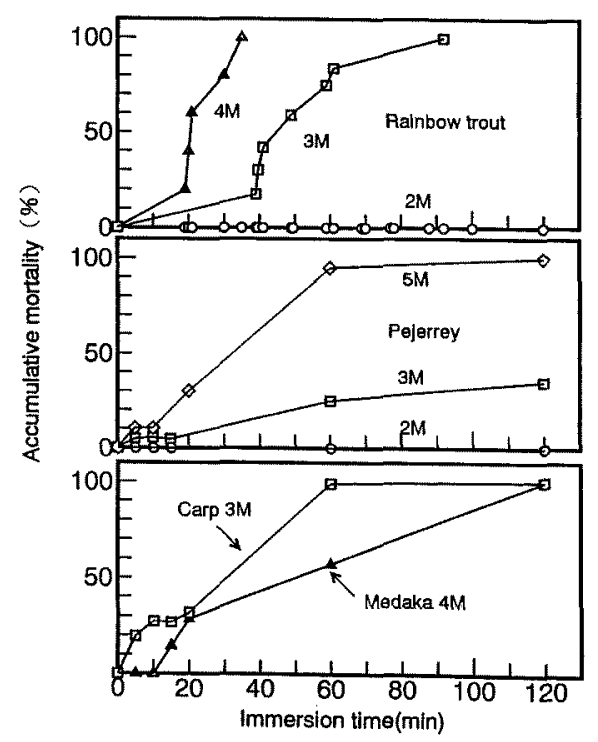

Fig. 2. Changes in the cumulative mortality of the embryos of four fish species during immersion in solutions of various DMSO concentrations; $O, 2 \mathrm{M} ; \square, 3 \mathrm{M} ; \triangle, 4 \mathrm{M} ; \diamond, 5 \mathrm{M}$. 
Figure 2 shows the relations between the cumulative mortality of embryos and the immersion time. The results clearly indicate that DMSO is harmful to every fish embryo although there are apparently considerable differences in tolerance among the different kinds of embryo. The mortality-time relationship for rainbow trout and pejerrey was tested using a solution of various DMSO concentrations as shown in Fig. 2. It is of interest to note that immersion in the $2 \mathrm{M}$ DMSO solution had no influence on the mortality of the rainbow trout and pejerrey embryos. However, when the DMSO concentration was more than $2 \mathrm{M}$, the mortality of most embryos quickly increased after the induction period in which the mortalities ranged from of 0 to $10 \%$. From the results for rainbow trout and pejerrey, it was found that the induction period reduced as the DMSO concentration of the immersion solution increased. This phenomenon is thought to be related to the inner embryo concentration of DMSO, as is described later.

\section{Average Lethal DMSO Concentration}

The DMSO concentration in embryos and cumulative mortality at identical immersion times were read from the uptake curves of DMSO into the embryos (Fig. 1) and the cumulative mortality-time curves (Fig. 2). The relationship between them is shown in Fig. 3. This figure indicates the direct toxicity of DMSO concentration on the fish embryos since the effect of the egg membrane resistance is eliminated. As shown in Fig. 3, the cumulative mortality of every fish embryo except for carp was $0 \%$ as long as the DMSO concentration in the embryo was less than about $0.7 \mathrm{M}$. This result indicates an upper limit of cryoprotectant. Data obtained by using the immersion solution of different DMSO concentrations are shown in each plot for rainbow trout and pejerrey, and are denoted by different symbols in Fig. 3. However, all plots tended to gather on an identical curve. This suggests that DMSO penetration into the embryo is restricted by the permeation rate of DMSO across

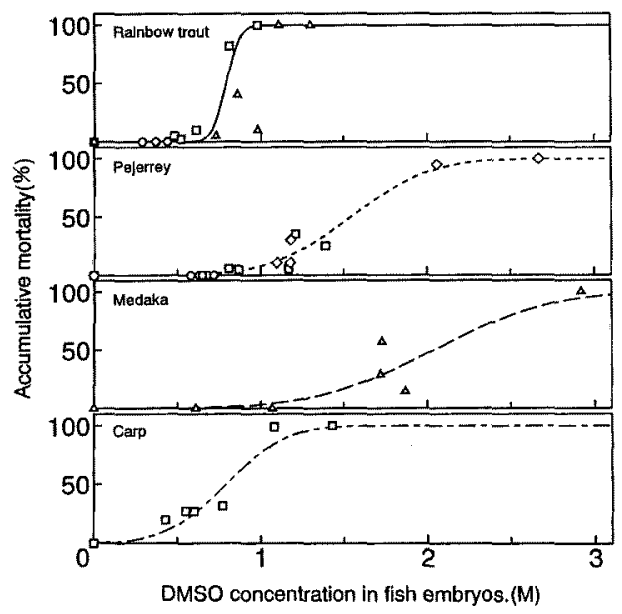

Fig. 3. Relations between the cumulative mortality and the inner DMSO concentration for the embryos of four fish species when immersed into solutions of various DMSO concentrations; $O, 2 \mathrm{M} ; \square$, $3 \mathrm{M} ; \triangle, 4 \mathrm{M} ; \diamond, 5 \mathrm{M}$.

The plots represent the actual measured data. The curves indicated by various patterns represent the fitted results to a cumulative normal distribution function. the egg membrane and the diffusion rate of DMSO in the embryos is very fast compared with the permeation rate. If the diffusion rate in the embryo is assumed to be low, as the initial difference of DMSO concentration becomes large between the outside and the inside of the egg membrane, the DMSO concentration should increase more quickly near the egg membrane, where the important organs are gathered. So, the lethal DMSO concentration averaged for the volume should become small as the DMSO concentration in the immersion solution increases. On the contrary, the result was independent of the DMSO concentration of the immersion solution. Therefore, the above assumption was disproved and the concentration gradient of DMSO in the embryo might be small.

The curves in Fig. 3 were obtained by fitting Eq. 2, which is a cumulative distribution function. Equation 2 assumes that the probability that the embryo dies depends on the DMSO concentration in the embryo as a normal distribution function:

$$
M_{\mathrm{A}}\left(C_{\mathrm{in}}\right)=50\left[1+\operatorname{erf}\left\{\frac{\left(C_{\mathrm{in}}-C_{\mathrm{av}}\right)^{2}}{\sigma^{2}}\right\}\right] .
$$

Where, $M_{\mathrm{A}}$ is a cumulative mortality as a function of the DMSO concentration $C_{\mathrm{in}}$ in embryos. $C_{\mathrm{av}}$ is the average lethal DMSO concentration corresponding to the concentration at which the cumulative mortality reaches $50 \% . \sigma^{2}$ is the variance and 'erf ( )' is an error function. As shown in Fig. 3, the relationship of the cumulative mortality and $C_{\text {in }}$ for every fish embryo is well approximated by Eq. 2. The parameters obtained from fitting, $C_{\mathrm{av}}$ and $\sigma^{2}$, are listed in Table 3. By using these results and the permeability coefficients in Table 2 , the cumulative mortality of each fish embryo for an arbitrary immersion time in a DMSO solution of any concentration can be determined from Eqs. 1 and 2.

The difference of $C_{a v}$ among the embryos reflects the difference in the tolerance of the fish embryo. It is generally known that the tolerance of fish embryos to chemicals depends on the development stage; embryos of the eyed stage are stable while those of the morula stage are relatively unstable. Arii et al. ${ }^{17)}$ reported that medaka embryo showed a difference in tolerance to DMSO depending on the development stage. In this report only the carp embryo was at the morula stage, while others were at the eyed stage. When comparing the eyed stage embryos, medaka embryos have a remarkable tolerance to DMSO, and their environment adaptability as a species is high since they showed a large variance $\left(\sigma^{2}\right)$. On the contrary, rainbow trout embryos were found to be very sensitive. Though it may be not valid to compare the tolerance of carp embryo to DMSO even with that of embryos of other stages, the average lethal DMSO concentration $C_{\mathrm{av}}$ of carp embryo was very small because the carp embryo was at the morula

Table 3. The lethal average DMSO concentration $C_{a v}$ and variance $\sigma^{2}$ of fish embryos

\begin{tabular}{ccc}
\hline Sample & $C_{\mathrm{av}}[\mathrm{M}]$ & $\sigma^{2}$ \\
\hline Rainbow trout & 0.792 & 0.005 \\
Pejerrey & 1.544 & 0.146 \\
Medaka & 2.039 & 0.308 \\
Carp & 0.790 & 0.081 \\
\hline
\end{tabular}


stage.

In summary, for several fish embryos, the tolerable inner embryo concentration of DMSO, i.e., the average lethal DMSO concentration, can be determined quantitatively, and depends on the fish species and the development stage. The tolerance to DMSO was higher in the order of carp, rainbow trout, pejerrey, and medaka. The obtained permeability coefficients of DMSO to the egg membranes were small because fish egg membranes are hard and thick. The relationship of cumulative mortality to DMSO concentration in embryos were well expressed by a cumulative normal distribution function. These results may be used to predict the cumulative mortality of each fish embryo at an arbitrary immersion time in a DMSO solution of any concentration. However, it should be noted that all results in this paper were obtained at a temperature which was close to room temperature. During cooling, freezing or immersion at different temperatures, our results may not be valid, i.e., the DMSO permeability to egg membrane and the lethal DMSO concentration might depend on temperature. The effect of temperature on permeability and lethal cryoprotectant needs to be investigated systematically.

Acknowledgements We wish to thank Dr. M. Hasobe, Dr. K. Sakai and Dr. C. A. Strussmann of the Department of Aquatic Biosciences for supplying the fish eggs, and also to Dr. T. Shirai, Department of Food Science and Technology for the helpful discussions on DMSO measurement. Au the above are affiliated with the Tokyo University of Fisheries.

\section{References}

1) Y. Haga: On the subzero temperature preservation of fertilized eggs. Nippon Suisan Gakkaishi, 48, 1569-1572 (1982).

2) B. Harvey and M. J. Ashwood-Smith: Cryoprotectant penetration and supercooling in the eggs of salmonid fishes. Cryobiology, 19, 29-40 (1982).

3) D. G. Wittingham and $H$. Rosenthal: Attempts to preserve herring embryos at subzero temperatures. Arch. Fisch Wiss., 29, 75-79
(1978).

4) B. Harvey: Cooling of embryonic cells, isolated blastoderms, and intact embryos of the zebra fish Brachydanio rerio to $-196^{\circ} \mathrm{C}$. Cryobiology, 20, 440-447 (1983).

5) J. Stoss and E. M. Donaldson: Studies on cryopreservation of eggs from rainbow trout (Salmo Gairdeneri) and coho salmon (Oncorhynchus Kisutch). Aquaculture, 31, 51-65 (1983).

6) X. S. Zang, L. Zhao, T. C. Hua, and R. Y. Zhu: A study on the cryopreservation of common carp Cyprinus Carpio embryos. Cryo Letters, 10, 271-278 (1989).

7) D. G. Whittingham: Low temperature storage of mammalian embryos, in "Proceedings of the Workshop on Basic Aspects of Freeze Preservation of Mouse Strains" (ed. by O. Muhlbock), Gustav Fischer Verlag, Stuttgart, 1976, pp. 45-55.

8) E. F. Graham, M. K. L. Schmehl, B. K. Evenson, and D. S. Nelson: Semen preservation in non-domestic animals, in "Artifical Breeding of Non-Domestic Animals, Symposia of the Zoological Society of London" (ed. by P. F. Watson), No. 43, Academic Press, New York, 1978, pp. 153-173.

9) J. H. S. Blaxter: Sperm storage and cross-fertilization of spring and autumn spawning herring. Nature, 172, 1180-1190 (1953).

10) H. Kurokura, M. Hirano, and M. Iwahasi: Cryopreservation of carp sperm. Aquaculture, 37, 267-273 (1984).

11) W. F. Rall and G. M. Fahy: Ice-free cryopreservation of mouse embryos at $-196^{\circ} \mathrm{C}$ by vitrification. Nature, 313, 573-575 (1985).

12) H. G. Hempling: Mass transfer of liquids across biological barriers, in "The Biophysics of Organ Cryopreservation, NATO ASI Series A" (ed. by D. E. Pegg and A. M. Karow, Jr.), Vol. 147, Plenum Press, New York, 1987, pp. 51-78.

13) T. Takashima and I. Hanyu: Suisanhansyokugaku, Ist ed., Midori Shyobo, Tokyo, 1989, pp. 184-185.

14) B. Harvey, R. N. Kelley, and M. J. Ashwood-Smith: Permeability of intact and dechroionated zebra fish embryos to glycerol and dimethyl sulfoxide, Cryobiology., 20, 432-439 (1983).

15) K. Arii, T. Suzuki, R. Takai, and T. T. Kozima: Tolerance of fish eggs to dimethylsulphoxide as the cryoprotectant. J. Tokyo Univ. Fish., 79, 121-126 (1992)

16) F. C. John and E. D. Patti: Quantity of dimethyl sulphoxide in solutions and tissues by high-performance liquid chromatography. Cryobiology, 28, 210-215 (1991).

17) N. Arii, K. Namai, and F. Gomi: Cryoprotection of medaka embryos during development. Zoological Sci., 4, 813-818 (1987). 